\title{
Performance without the Pain, Extending the Capabilities of Low-Maintenance Home-Lab Systems for Biological Crystallography.
}

Matthew M. Benning ${ }^{1}$ and Michael Mrosek ${ }^{2},{ }^{1}$ Bruker AXS Inc., 5465 East Cheryl Parkway, Madison, WI, 53711 USA, ${ }^{2}$ Bruker AXS GmbH, Oestliche Rheinbrueckenstrasse 49, 76187 Karlsruhe, Germany.

The performance of home-lab x-ray systems for macromolecular crystallography has increased substantially in the last decade. However the performance usually comes with a high price creating the dilemma: find the resources to keep the home-lab instrument running or collect all your data at the beamlines. The Bruker D8 VENTURE has two new improvements in source and detector technology that provides great performance without high operational costs. The new air-cooled I $\mu S$ DIAMOND microfocus sealed tube source uses a unique anode technology, diamond hybrid, to produces intensities similar to modern rotating anodes. The anode consists of a diamond substrate coated with copper. Properties of diamond such as high thermal conductivity, low thermal expansion and extreme hardness make it an ideal substrate allowing not only higher power loading but greater long-term stability of the source. The PHOTON III is a new CPAD (charge-integrating pixel array detector) which utilizes a mixed-mode approach for data collection. The weak reflections are measured in photon-counting mode and the strong reflections are measured in the proven integrating mode. This allows the PHOTON III to have the ultrasensitivity to collect very weak reflections while not suffering from charge sharing or nonlinearity effects common to other photon-counting detectors. The hardware advances along with experimental data will be discussed. 